\title{
Author Correction: Contrasting responses of autumn-leaf senescence to daytime and night-time warming
}

Chaoyang Wu Di , Xiaoyue Wang, Huanjiong Wang (D), Philippe Ciais (D), Josep Peñuelas (D), Ranga B. Myneni, Ankur R. Desai D, Christopher M. Gough, Alemu Gonsamo (D), Andrew T. Black, Rachhpal S. Jassal, Weimin Ju, Wenping Yuan, Yongshuo Fu, Miaogen Shen, Shihua Li, Ronggao Liu, Jing M. Chen and Quansheng Ge (D)

Correction to: Nature Climate Change https://doi.org/10.1038/s41558-018-0346-Z, published online 26 November 2018.

In the version of this Letter originally published, there were errors in Fig. 1a. The sites denoted purple were described in the legend as ' $P_{\text {day }}>0.05 \& P_{\text {night }}>0.05$ ', but should have been labelled ' $P_{\text {day }}<0.05 \& P_{\text {night }}>0.05$ '. The sites denoted green were described in the legend as ' $P_{\text {day }}>0.05 \& P_{\text {night }}>0.05$ ', but should have been labelled ' $P_{\text {day }}>0.05 \& P_{\text {night }}<0.05$ '. The sites denoted orange were described in the legend as ' $P_{\text {day }}>0.05 \& P_{\text {night }}>0.05$ ', but should have been labelled ' $P_{\text {day }}<0.05 \& P_{\text {night }}<0.05$ '. These errors have now been corrected.

\section{Publisher Correction: Contrasting responses of autumn-leaf senescence to daytime and night-time warming}

Chaoyang Wu D , Xiaoyue Wang, Huanjiong Wang (D), Philippe Ciais (D), Josep Peñuelas (D), Ranga B. Myneni, Ankur R. Desai D, Christopher M. Gough, Alemu Gonsamo (D), Andrew T. Black, Rachhpal S. Jassal, Weimin Ju, Wenping Yuan, Yongshuo Fu, Miaogen Shen, Shihua Li, Ronggao Liu, Jing M. Chen and Quansheng Ge (D)

Correction to: Nature Climate Change https://doi.org/10.1038/s41558-018-0346-z, published online 26 November 2018.

In the version of this Letter originally published, the author Andrew T. Black was mistakenly denoted as being affiliated with the Institute of Geographical Sciences and Natural Resources Research. His affiliation has now been corrected to: Faculty of Land and Food Systems, University of British Columbia, Vancouver, British Columbia, Canada. 